\title{
IOT BASED SMART HOME ASSISTANT
}

\author{
${ }^{1}$ Saajan Kumar Jha, ${ }^{2}$ Nishit Jain, ${ }^{3}$ Mukund Badatiya, ${ }^{4}$ Dr. Vinayak Shinde \\ ${ }^{1,2,3}$ Student, ${ }^{4}$ Head and Associate Professor, Computer Engineering, Shree L.R Tiwari College of \\ Engineering, Mumbai, India, ${ }^{1}$ saajanjha1998@gmail.com, ${ }^{2}$ nishitjain0987@ gmail.com, \\ 3 mukundbadatiya@gmail.com, 4 vdshinde@gmail.com
}

Abstract - The world is full of automation and the people living in this world are surrounded with automated systems. Industries, business sectors, even homes are being automated. Operating various home appliances conveniently along with saving energy are the prominent factors possessed by Smart Home. A quick increase is seen in people's needs in order to develop the social economy of appliances present in a house. If we look at the existing system, we have Google home and Amazon echo which provide functionalities such as switching on/off appliances remotely from anywhere. This paper primarily focuses on a smart home system which allows users to control any electrical appliance using their smart phone google assistant. Apart from this the system also helps in keeping a track on air quality, sound and light intensity, temperature and humidity of home. It also provides analysis of the aforesaid parameters using various graphical methods. It starts the appliance itself on detecting human motion and notifies the user about the same so in case if there is an intrusion the user will come to know about the same. It also takes care of user's safety as it informs about gas leakage and fire detection preventing any major damage. The need for integration is seen in society, as the idea of a Smart home system is still a new research area. Convenience, security, time management, and connectivity act as prominent factors to motivate and build a system like Smart Home. This paper hence provides a detailed inspection on the architecture, procedures, technologies required for a smart home system. The best protocols and technical solution acquired in the IOT Based Smart Home Assistant are discussed in the paper.

Keywords - Adafruit.io, Android, Automated Appliances, comfort, Firebase, Google assistant, IFTTT (IF This Then That), IoT, MP Android chart, MQTT (Message Queue Telemetry Transport), security, sensor system integration.

\section{INTRODUCTION}

An association of technology and services is involved in smart homes. Smart Home promotes an advancement towards home technology. A straightforward definition for home automation is that the ability to try to do tasks mechanically and monitor or amend status remotely [7]. Ideally, this concept has a very vast scope in future. This concept is still on the verge of advancements, these key features will act as a pathway towards the introduction of the idea. Home security has always been a major concern throughout the world. Hence, with the smart home application, the user will get a quick notice about the intruder, if it detects fire or a gas leakage. So, our smart home security system bridges the gap between the problems and solutions to it.

A smart home permits the whole home to be machinecontrolled and thus offer ease and convenience to everyday activities within the home. Versatility, Reasonability and Portability defines the system [8]. In the near future, it will be very beneficial for all the electronic devices which will be able to take the advantage of this technology. IOT has become one of the buzzwords and it has an immense contribution in the field of security [4]. There is a high misconception of this technology being a pure networking concept. But this technology is much more than that, it relatively helps to ease the workload. The government of India has lately announced to develop more than 100 cities which have demand for IOT and smart appliances. As everyone seems to be conversant in smart phones and google assistants, this model provides the feature of controlling the electrical appliances through google assistant from anywhere [4]. As we tend to understand safety is given the highest priority during this paper a fire detection system [6], a gas detection system [1] and an intrusion detection system has been an addition to this model [2],[3]. The system uses google firebase for storing sensor values on a real time basis [5].

\section{PROBLEM STATEMENT}

- $\quad$ "To develop a system where user can monitor and analyze their home based on various parameters and can control it's appliances using google assistant using Internet of Things (IOT)"

- $\quad$ To achieve this, we will be using an IFTTT cloud server which will trigger our controller on receiving voice command. 


\section{SCOPE AND MOTIVATION}

The thinking behind building this product is to control the household appliance remotely through our mobile phones. Our paper also focuses on control of various devices of the house with the internet. To improve the security in extremely restricted areas [3]. It can be used for a long time in the future. Using our project, users can monitor parameters such as air quality, light intensity, sound intensity, temperature and humidity [4],[11]. The system can detect events such as motion, fire and gas leakage [1]. It is limited to appliances having less power requirements. We can manage all the home appliances or devices from anywhere in the world. To keep all the technology together in your home through one interface [9]. The concept is evolving as home automation in the classic sense meets an increasing range of products.

The motivation for developing sensible home systems comes from many reasons, however the most prominent reasons are convenience, security, energy management, property and luxury. In today's world IOT is a huge thing. Today's generation wants everything quicker and work to be done on one click [8]. IOT is the new era of technology and is changing everyone's lives in some ways, habits and business. Convenience is another way of claiming "time saver", and in today's world everything is moving quicker, each second has a price. We tend to additionally believe in the convenience of human life and safety. Sensible homes scale back human efforts also as scale back power consumption. Having a smart home can change lives of disable peoples also. Having a smart home, the disable people can have a trust factor of a security [3]. Security is additionally a giant considering the emergence of sensible home systems. With a complicated enough system, home security becomes a strong tool that offers peace of mind and power to the user.

\section{PROJECT OBJECTIVES}

- To identify various objects in our home which are possible to monitor and manage using our project.

- Identifying various sensors required for the objects listed out in objective 1 .

- To select suitable sensors for our project from various sensors and actuators identified in objective 2 .

- The monitoring of the house is done based on various sensors used and appliances connected to the system. Users can use the application to interact with the system.

- To create a system where users can control it's appliances using google assistant”.

- To achieve this, we will be using an IFTTT cloud server which will trigger our controller on receiving voice command.

- To study various development boards available in the market and selecting the most suitable one as per our requirements.

- To study various cloud platforms available in the market.

- To select suitable cloud platforms based on project requirement.
- To select a proper database management system related to our project requirements and convenience.

- To study various database management systems.

- Identifying different attributes of objects listed out which will be considered while creating the database.

- To identify various tools, languages and platforms available for creating applications.

- To select appropriate tools, language and platform for creation of application.

- To identify various methods for triggering controllers through google assistant.

- To select a suitable method based on our project requirement.

- To identify various methods for sending signals to the controller through Google assistant.

- To provide access to authorized users.

- To provide an enticing interface to users for monitoring home based on various parameters and for monitoring as well as controlling appliances.

- To identify various methods for analyzing data.

- To select suitable methods for data analysis.

- To notify if there's any intrusion noticed.

- To ensure safety by notifying users about fire detection and gas leakage.

- To view sensor value from anywhere in the world.

- Live monitoring of all sensors and appliances from anywhere.

- To minimize the operating cost and improve the comfort.

- To provide improved convenience, comfort, energy efficiency and security.

- To increase quality of living, especially of the elderly and the disabled.

- To reduce the power consumption.

\section{LITERATURE REVIEW}

In, Gas leakage detection: This paper proposes a design in which the user can automatically book a cylinder gas when the gas is about to get empty. It sends the notification to the gas agency with the help of Wi-Fi. In addition, they have also built up a system with the help of a gas detection sensor where the user is notified about the gas leakages through the alert notification or by a call. In this paper they have used an MQ-5 sensor to detect the gas leakage, which can sense the H2, LPG, CH4 and CO. So, we expected that this sensor could be more useful as it notifies the alert message and can be used for safety means [1].

In, Intrusion detection system: During this paper, the passive infrared device (PIR) is employed to detect motion. This paper discusses the application of PIR sensors in such systems and use of ZigBee to create a wireless device network and ESP8266 module to send data to a foreign server. PIR sensor, being passive in nature is undetectable and works well within the dark surroundings conjointly. So, we decided to use PIR Motion sensors to detect if any intrusion has been detected. We have also added the 
notification message or alert message given to the user if intrusion is detected. It is used for safety purposes [2].

In, PIR Based Human Detection System: During this paper, we tend to area units providing home security for the theft by implementing sensible closed-circuit television mistreatment RP and PIR sensors. Now-a-days, the IoT plays a serious role in several fields by automating the appliance. The camera and PIR detector area unit integrated in such the simplest way that any movement within the area, switches ON the camera mechanically. So, in our case the house owner will get an alert notification the projected work provides a smart home automation system for intrusion detection [3].

In, Voice Control Personal Assistant device: This paper proposes a design of IoT-based smart home application with a remote-control device that is developed using voice commands for disabled people. Smart home control systems help disabled people to control their home electrical devices such as television (TV), lights, and fans using only voice commands without moving to turn on or turn off electrical equipment. The voice recognition on electrical equipment is using the Google Assistant's application on smartphones. The Google Assistant application will accept voice commands when the pronunciation is correct. We expect that this device could be more useful to help disabled people interact with their environment by utilizing IoT technology facilities [4].

In, Google Firebase: This paper styles an online of Things system (called Just IoT) that is especially divided into four parts; back-end Google base period info, front-end SPA (Single Page Application) internet program (including mobile watching App), controller software-hardware, and intelligence server that support MQTT affiliation and condition management. Users will build applications within the top of the system, to serve customers, and to run a business. The event of information modification of base info can trigger Angular's two-way information binding to attain Three-way information binding result to implement serverless architecture simply. the info in base info is scanned and written by the front-end devices (web apps, mobile apps, and controllers) directly. The intelligence server is an associate degree MQTT server that supports the connections of comparatively weak embedded controllers like the Arduino controller. The intelligent server may be considered as associate degree negociant between the base period database and weak controllers, that performs the transfer of data and remote commands [5].

In, Fire Detection System: The fire detection could be a study interest for the house police investigation services. This paper presents the intelligent hearth push notification service supported camera and internet application server with real time processing much for the mobile user in a good home. We tend to support an automatic hearth identification supported the camera image and to alert the hearth situation, and to warn the mobile user consequently. Also, we tend to appraise the interval of the hearth detection and push notification to verify the performance of the projected service. The projected hearth push notification service is meant to enhance the house police investigation in terms of a sensible mobile approach for the protection home environments [6].

Smart Home Automation: Advancement in IoT primarily based application has become the state-of-the-art technology among the investigators because of the supply of net every place. During this paper, a good energy economical home automation system is planned that may access and manage the house equipment from each corner of the planet. For wireless property, the static information processing address is employed. Home automation on multimodal applications that may be operated by mistreatment voice recognition command of the user mistreatment the Google Assistant or through an online based application. So, the main objective of this work is to form our home automation system safer and intelligent [7].

In, IOT and its application: We are entering in a new phase of computer technology called IOT. Internet of Things (IoT)devices are used as remote or virtual laboratories. This paper proposes how the smart machines are connected to each other and interact or communicate with each other. Especially, these usage eventualities can implement a learning strategy for every downside to be solved. The present work shows the evolution of those archetypes and their application within the teaching of disciplines/subjects defined in engineering, like distributed computing and cyber security [8].

In, IOT With Blynk App: This paper embodies practicality of node esp8266 square measure connected with either on top of given house application like fan, light, water pump, husbandry with ease of coding and hosting on-line with internet server. All the practicality is handled by Mobile App created in golem application, from which house application square measure controlled with facilitation of net. This paper is informative about the observation of circuit devices through wireless using Node MCU and monitoring of appliances through an application. According to the need it can connect various devices, sensors and appliances [9].

In, MQTT Broker: IOT- Internet Of things is nothing but a communication network which is used to share data between two different devices. In this paper they have told us that MQTT Broker is a common application layer protocol which is used to share information in IOT devices. So, we decided to experiment some of the works from this paper in the project to send commands from one device to another [10].

Power consumption using LDR Sensor: This paper describes one applicable technique to optimize the wattage consumption using lightweight Dependent resistance (LDR) 
sensing element. The LDR sensors interface with each other, and therefore the necessary intensity needed by the bulb can be calculated and therefore the bulbs can offer the desired lumen for the surrounding/workspace. The output would be full of surrounding lightweight sources. So, this system will offer optimized intensity for the encircling and reduces the wattage consumption throughout daytime [11].

\section{PROPOSED SYSTEM}

The project consists of different modules which work individually and is integrated as a whole in order to make one complete system, as a result the proposed system is divided into three different modules namely a software module, a hardware module and a module where the appliances can be controlled through the Google assistant.

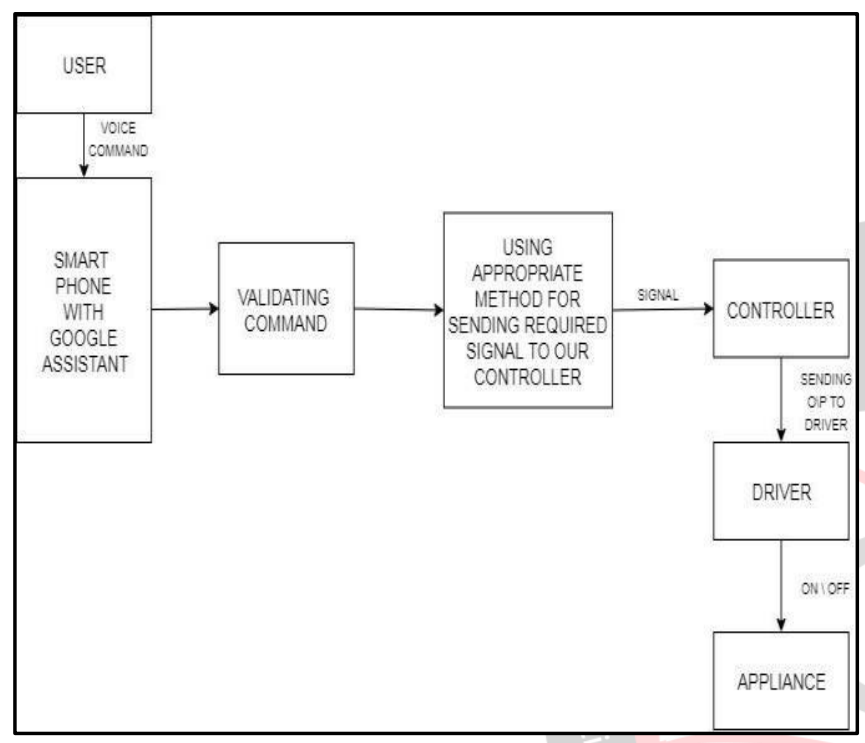

Figure.1: Proposed System for controlling appliances through Google assistant

The above system proposes how the user can control the appliances through a voice command using google assistant. According to the proposed system the user needs to give a voice command to the google assistant the command is validated; any invalid command will give a negative acknowledgement to the user [4]. Once a valid command is said the appropriate method which in this project case is IFTTT and MQTT Broker will be used to send the signal to the controller [10]. The driver will be activated once the controller sends the signal to the driver which in turn executes the command said.

The above system is a proposed hardware module for the project. According to the proposed system, the controller has an input channel and an output channel, it also includes a Wi-Fi module along with an external power supply [7],[8]. All the sensors namely motion, temperature, humidity, fire, gas, sound intensity, light intensity pass all its sensor value to the input channel, the output channel will be used to control the appliances. Our controller requires an inbuilt Wi-Fi module to connect to the MQTT Broker through the gateway [10].

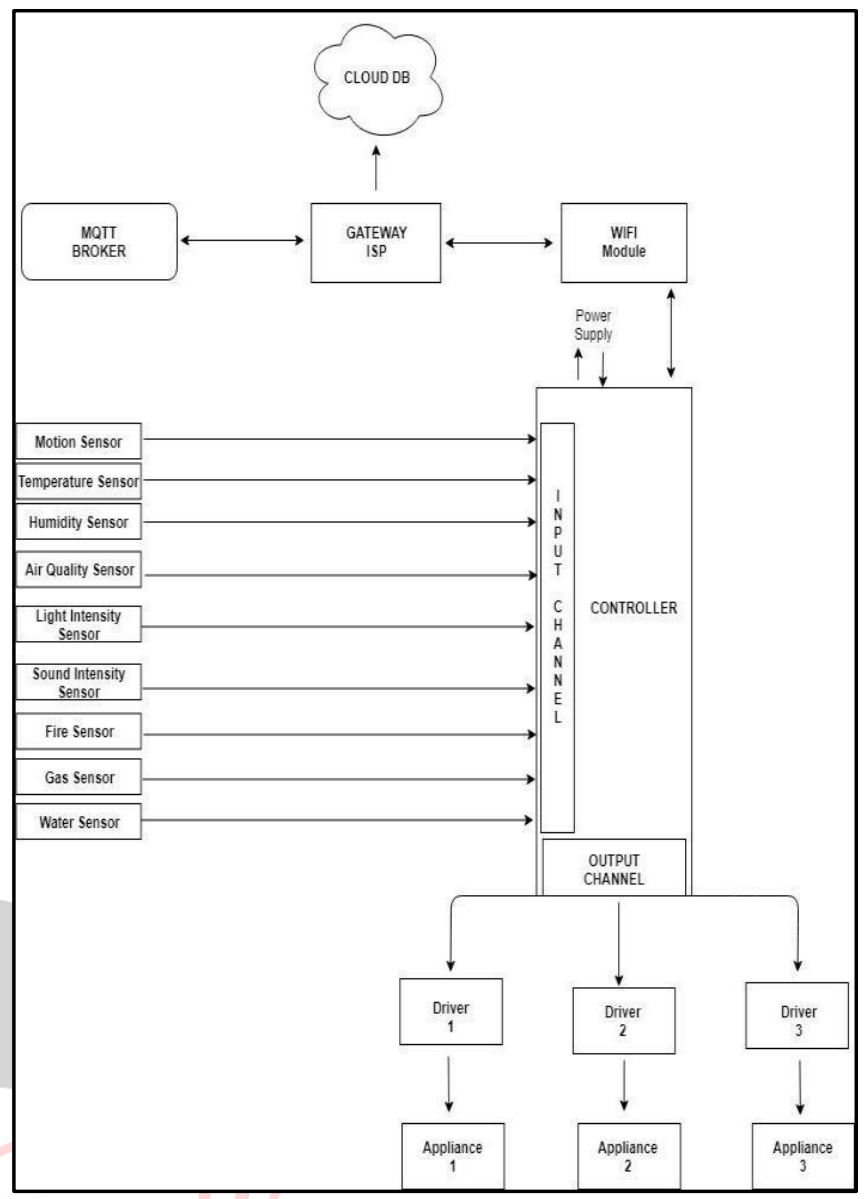

Figure.2: Proposed System for hardware module.

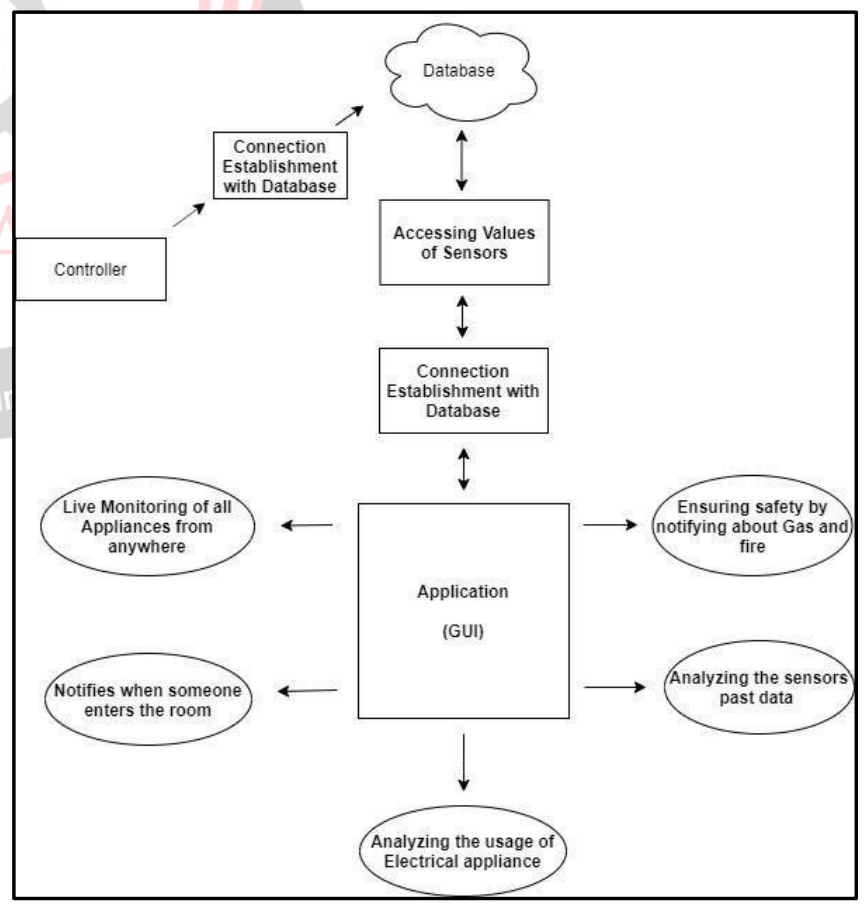

Figure.3: Proposed System for software module

The above system is a proposed software module for the project. The proposed system depicts the total software module of the project. The software module consists of an android application, through this application the user can monitor all the appliances and can also get all the sensor values [9]. The user can monitor all the appliances through the application. The user will also receive a notification if 
any of the incidents such as intrusion, gas leakage or fire breakage occurs. The controller sends all the values to the database on a real time basis, all these values can be monitored by the user once the user login to the android application.

\section{SYSTEM ARCHITECTURE}

Figure 4 is the architecture which shows how a user can control the appliances using the google assistant. In order to achieve this, we have to create applets on our IFTTT server. The applet creation involves two entities which in our case are Google Assistant and MQTT Broker. Whenever the if condition is satisfied by entity one, a defined action is performed by the IFTTT for entity two. In our project if we say the custom created command to our google assistant then the IFTTT will send the feed to our MQTT broker [10]. The actual flow of this part goes like. The user gives the voice command to the google assistant using their smartphone, the command can be anything say to switch on the light, as soon as the command is given a connection with the IFTTT is established after connection establishment the command will be validated, if the command is invalid negative acknowledgement is given to the user. If the command is a valid one the system is connected to the MQTT broker and the command feed is given to the MQTT broker, once the feed is given a connection is established with the MQTT client which in turn triggers the controller[7]. The controller signals the driver of the appliances then according to the command it reacts to the appliances [4]. When the connection is established with the MQTT client at the same time there is also a database connectivity which stores the timestamp of the given command.

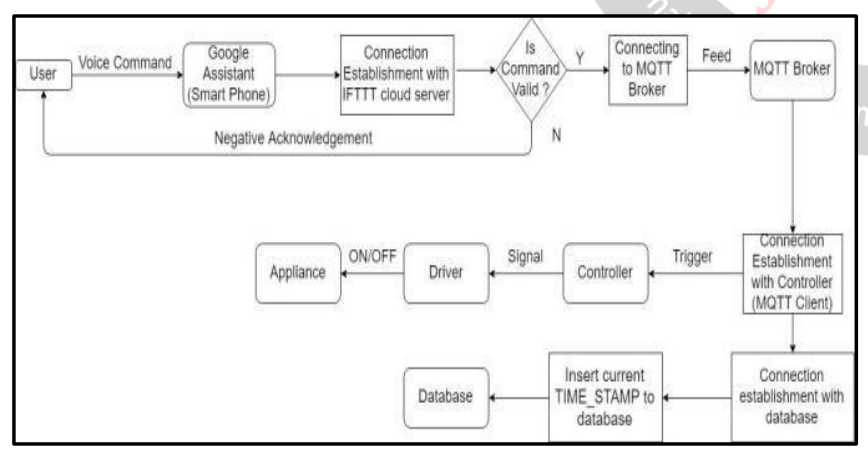

Figure.4: Controlling Appliances through google assistant

Figure 5 is the architecture which shows how the user can monitor its household appliances and the whole system through the android application [9]. The user needs to login to the application with the help of its login credentials, if the user is new the user needs to register for the product. The user is authenticated with the help of firebase authentication. The user will find many options, one of which is that the user can monitor its appliances. Since our Controller is subscribed to our MQTT Broker, the appliances operate as per the current data of their respective feed [10]. Whenever there is a change in the feed the controller triggers the relay driver and updates the field in the firebase database [5]. As the value is updated in the database our application which has event listeners implemented on every appliance field changes the status of the appliance. The application also provides an interface where the user can request to add or remove an appliance to the system, provide any feedback or report any problem. The response of the user is stored in the database which is later retrieved by the admin [5].

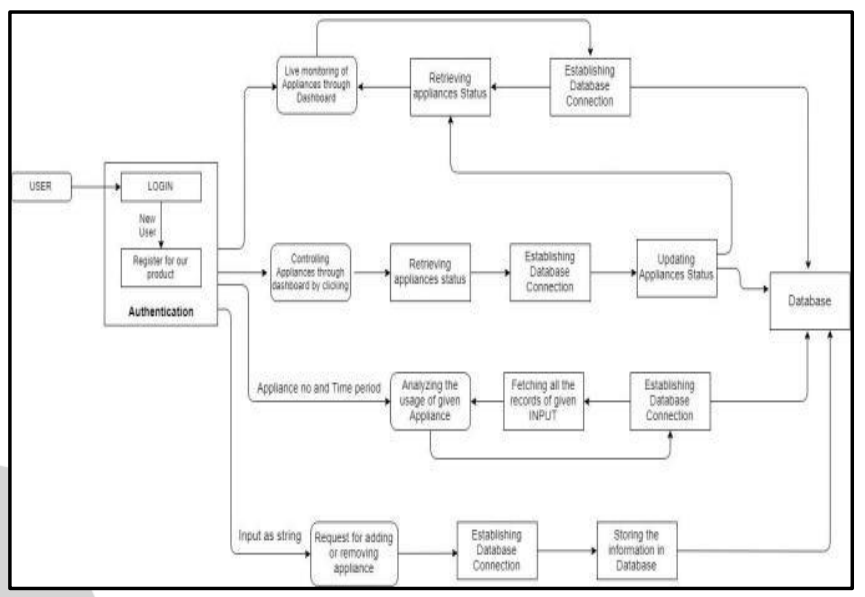

Figure.5: Monitoring of appliances and user interaction with admin

Figure 6 is a module which proposes how the system works if an event such as motion, gas leakage or fire breakage is detected. If a motion is detected by the sensor(PIR motion Sensor)[2], it writes to the output pin, a connection is established with the database, and the timestamp on when the motion is detected is directly stored in the database by the controller[5]. At the same time the sensor checks the status of the appliances if the appliances are off it eventually switches the appliances on, the timestamp of this event is also stored in the database. Similarly, if a gas leakage or fire breakage is detected by the sensors (MQ-6 and flame sensor) [1], a connection is established, and the timestamp of the event occurred is stored in the database [5]. Now, if any of the event is detected a notification is pushed by the android application through which the user can analyze the event detected[9].The notification also has additional features to check with neighbors or directly contact the emergency number..

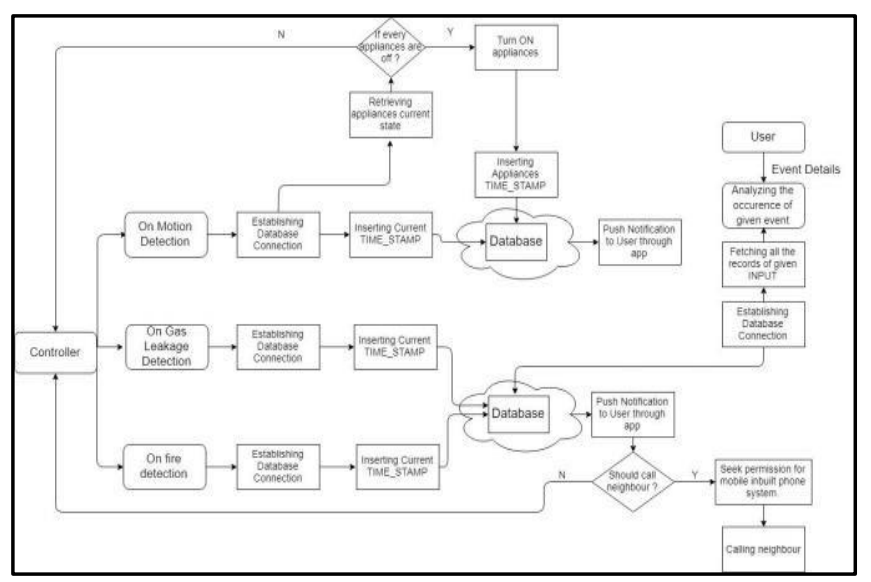

Figure.6: Architecture of Event detection Module 
Figure 7 is the architecture which proposes how the user can analyze the value of analog sensors i.e. Light and sound intensity, Air quality, humidity Room temperature. The light intensity sensor (LDR sensor) and sound Intensity sensor (sound module sensor) [11] constantly senses all the intensity of the household and stores all the sensor values in the database on a real time basis. Similarly, the room temperature and humidity sensor (DHT 11 sensor) and the air quality sensor (MQ-135 sensor) [4] constantly stores the sensor value in the database. The values of these sensors are stored by the controller periodically in the database and the real time values are stored on the different individual fields which are updated in real time. The analysis of these data is done using graphical methods with the help of MPAndroid Chart [9].

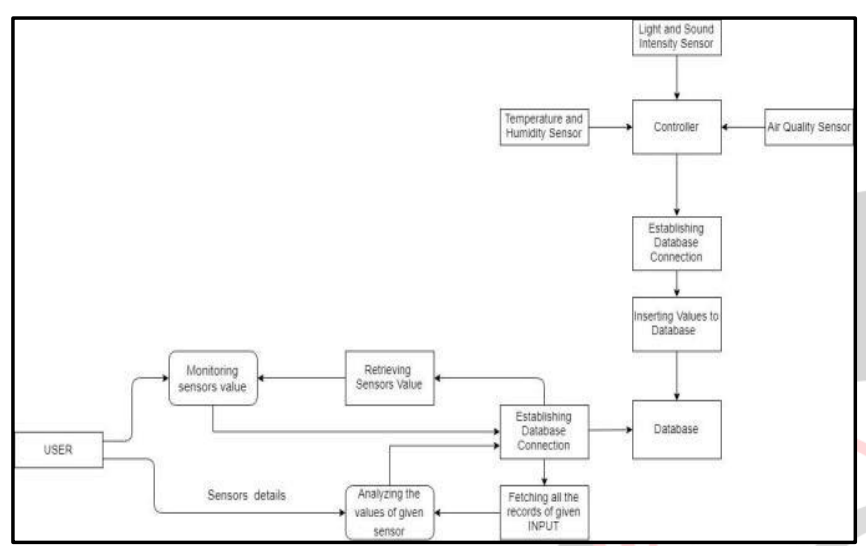

Figure.7: Architecture for Analysis of analog sensors

\section{RESULT}

By using the methodologies mentioned above we achieved the following results in our project. Referring to the system architecture in Figure.4, we were able to control our appliances using google assistant with the help of IFTTT server and Adafruit.io (MQTT Broker).

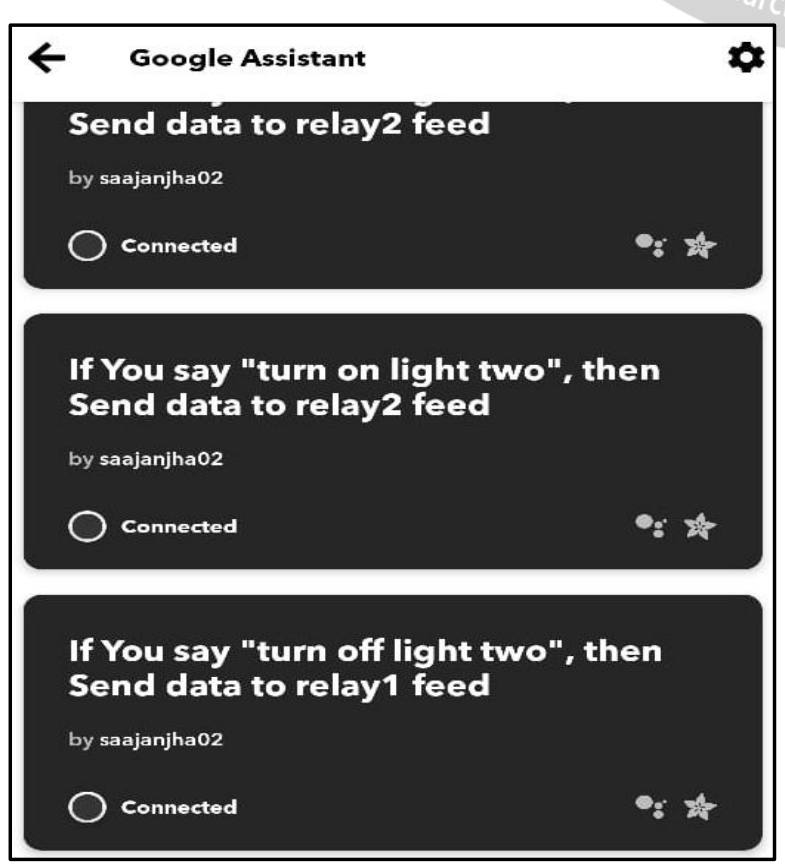

Figure.8: Applets creation on IFTTT
Figure.8 refers to the part of applets creation on IFTTT, which sends the data to our MQTT broker when a valid command is said. For example, when the user will say "turn on light two" it will send the data to the corresponding feed.

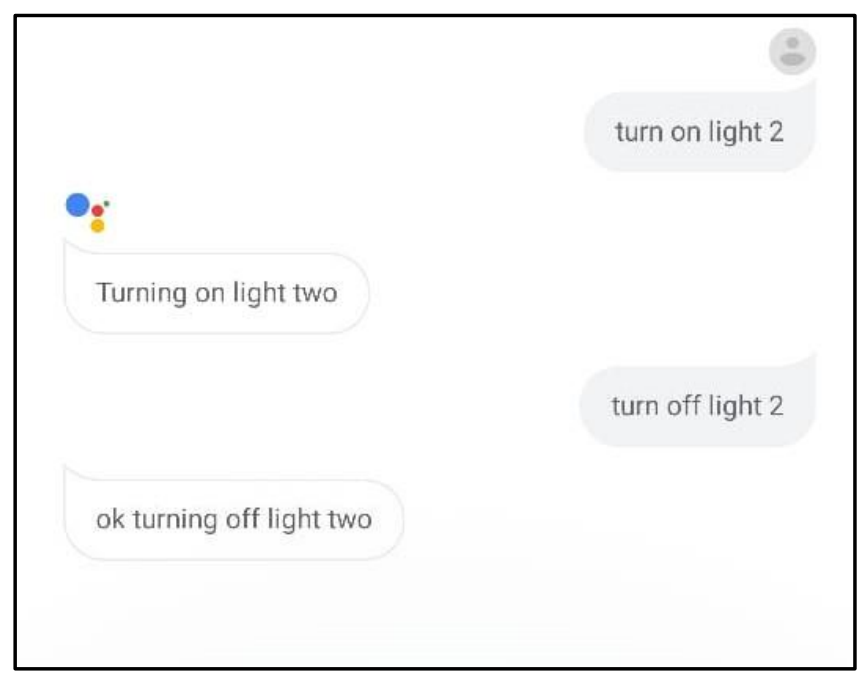

Figure.9: Acknowledgement by google assistant

Figure.9 shows the acknowledgement received from the google assistant when we control our appliances with it. Once the data is received by the MQTT Broker, our controller which is already subscribed to it triggers the required driver. Thus, controlling the appliances as per required. The android application is a major part of the project as it acts as an interface to the user. The user can login to the android application and it will provide the user its profile. Through the application the user will be able to analyze it's home based on various parameters, monitor the appliances and get notified with all the warnings that may occur in its household. Below are the screenshots of the application.

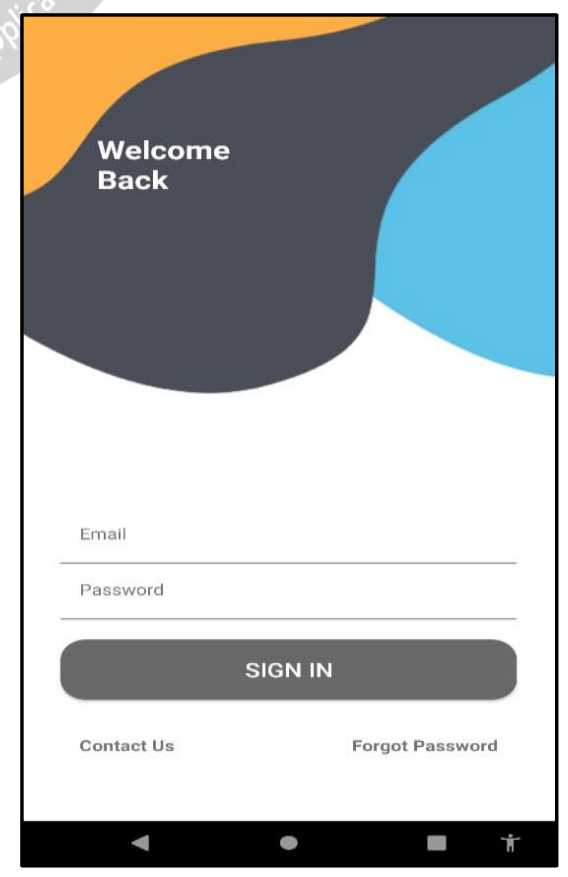

Figure.10: Login Page

The above figure is the login page of the android 
application where the user needs to enter its login credentials and after verification the user can use the application.

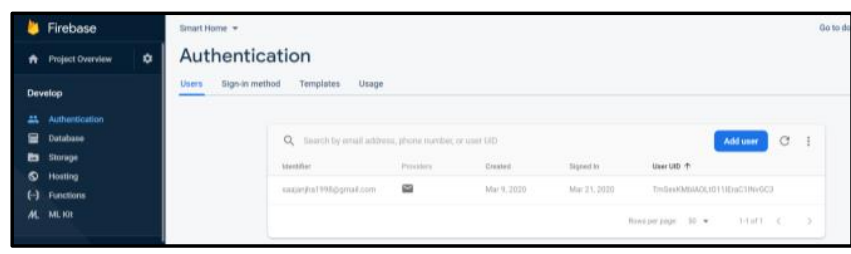

Figure.11: Firebase Authentication module

Figure.11 shows our firebase authentication module. The verification is done using firebase authentication, where we can keep records of all our users.
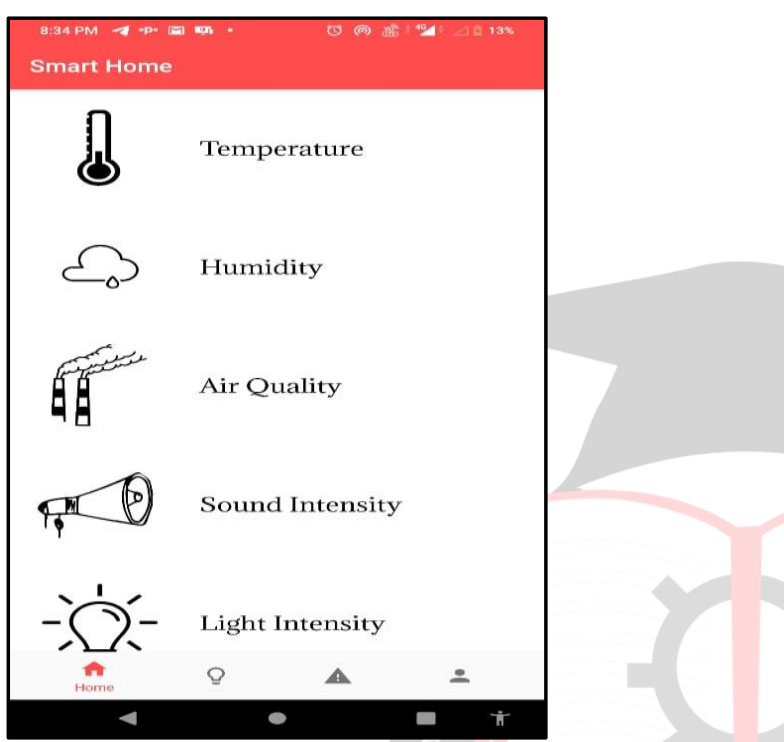

Figure.12: List of parameters for live monitoring and analysis.

The above figure shows the home page of the application which exhibits all the parameters for live monitoring and analysis. Users can select any parameter and view the live value and data analysis.

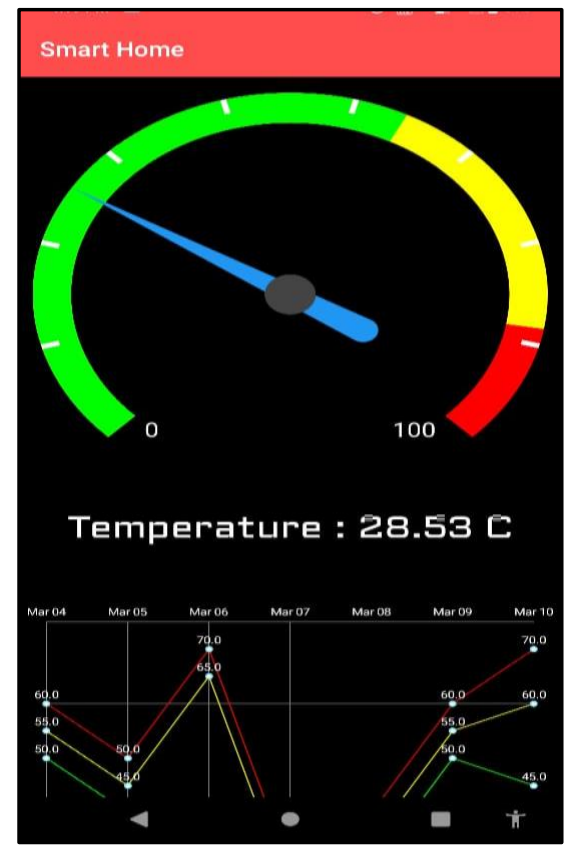

Figure.13: Live Monitoring of Temperature
Figure.13, shows the live monitoring of temperature at the user's home. The sensor updates the value in the database at regular intervals and the same change is reflected on the gauge by setting it to the updated value.

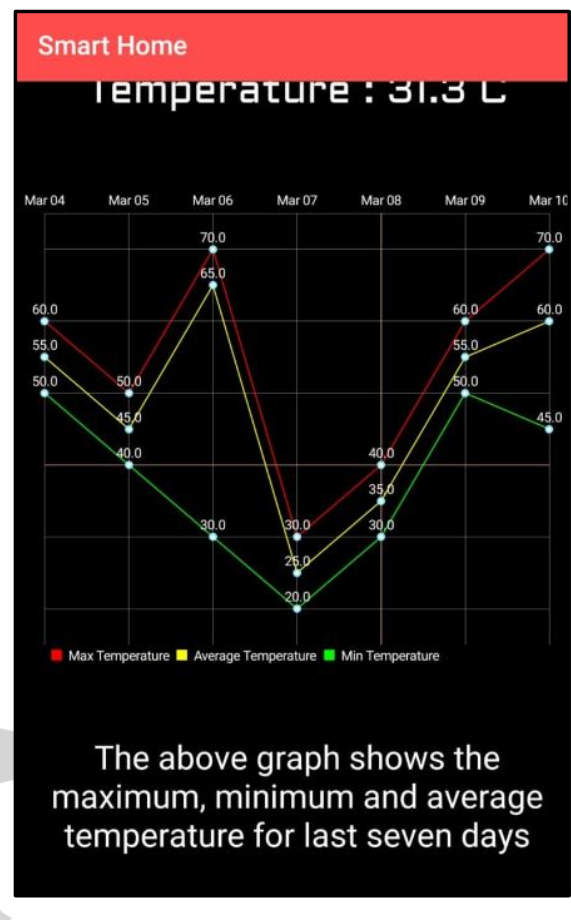

Figure.14: Data Analysis Part (Line Graph)

Figure.14, shows the data analysis part using line graph method. The line graph is created using the MPAndroid chart library. The line graph shows the min, average and max value of the parameter from the last seven days.

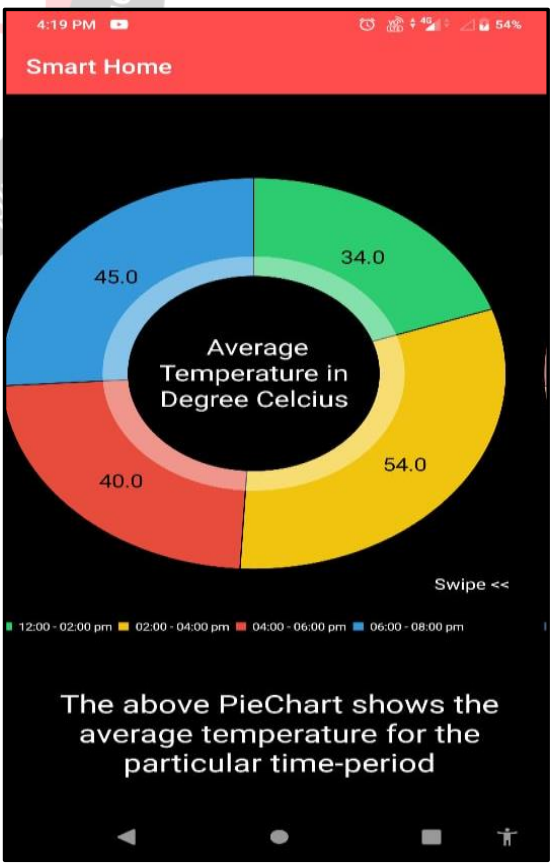

Figure.15: Data Analysis Part (Pie Chart)

Figure.15, shows the data analysis part using the pie chart method. The pie chart is created using the MPAndroid chart library. The pie chart shows the average value of the parameter for a given time slot. 


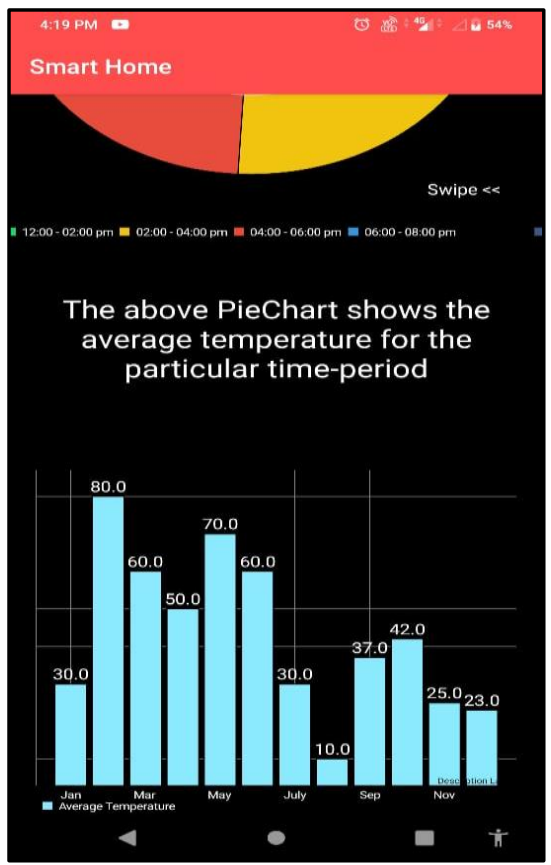

Figure.16: Data Analysis Part (Bar Graph)

Figure.16, shows the data analysis part using bar graph method. The bar graph is created using the MPAndroid chart library. The bar graph shows the average value of the parameter for a given month.

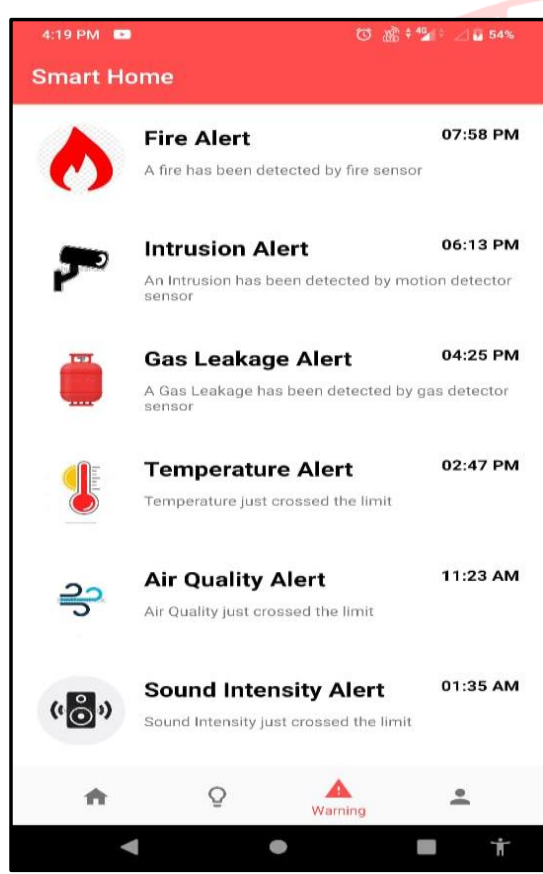

Figure.17: Warning Page

Figure. 17 shows the warning page of the app. It has all the previous and new warnings or alerts generated by the system. When any incident occurs like intrusion, fire, rise in temperature or air quality. The controller adds the event in the database and post that an alert notification is generated from the app as shown in figure 18 .

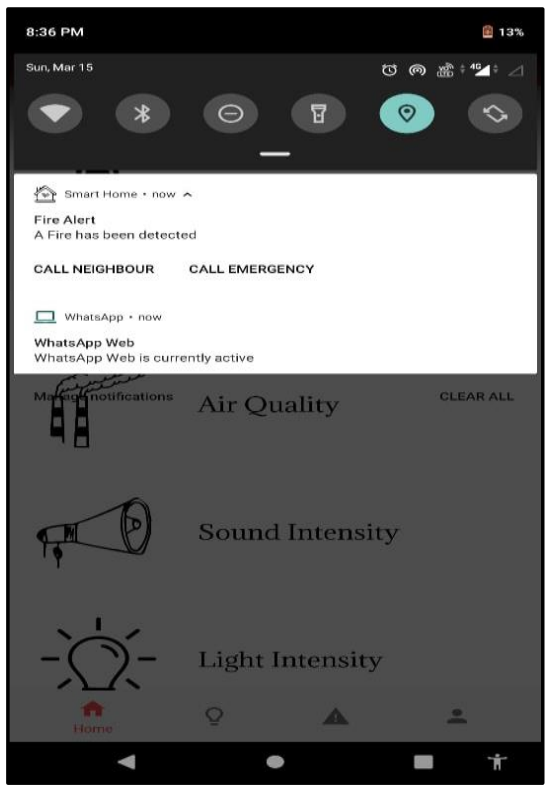

Figure 18 Fire Detection notification to user

The above figure 18 is the alert message generated when a fire leakage is detected in the house. The alert has additional functionalities whether we want to check with our neighbor or directly call the emergency number.

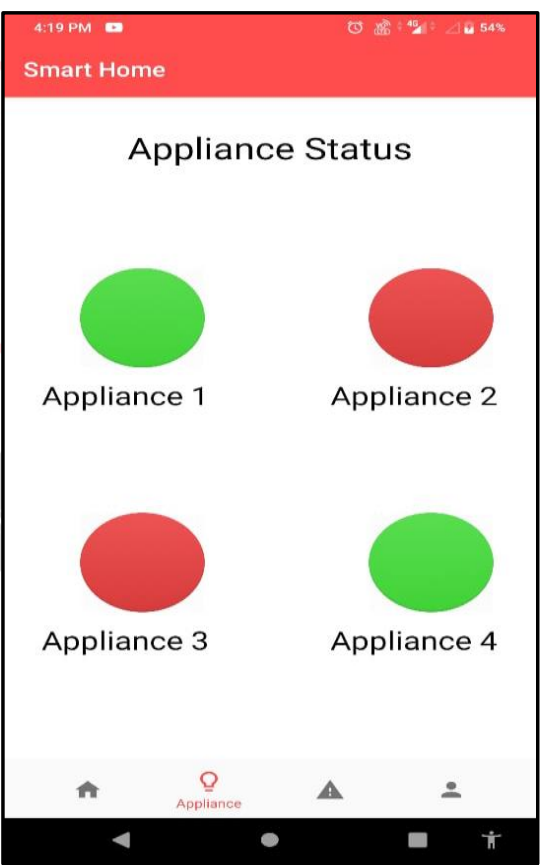

Figure.19: Appliance Section

The above figure shows the appliance section of the app, where the user can see the live status of the appliances. Whenever the controller triggers the appliance, the field in the database is updated, and post that the status in the app is updated. 


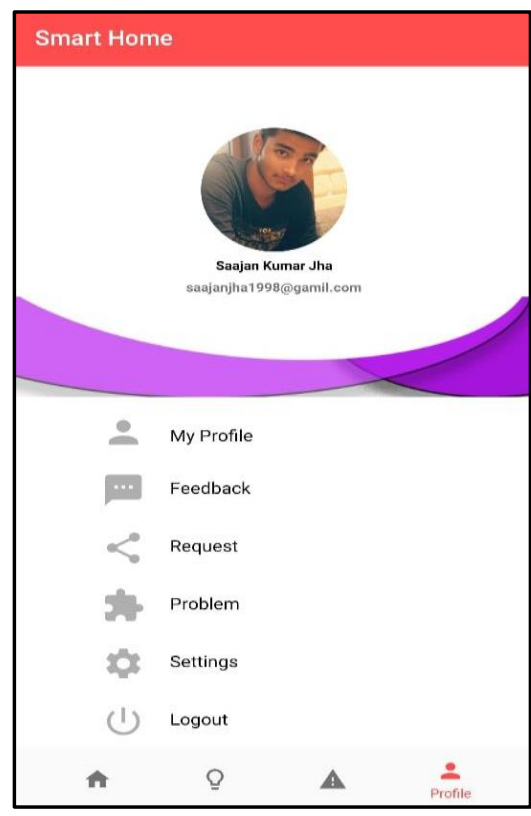

Figure.20: Profile Section

The above figure is the profile section of the user where the user can provide feedback, can request about adding removing any devices or parameters or report any problem they're facing.

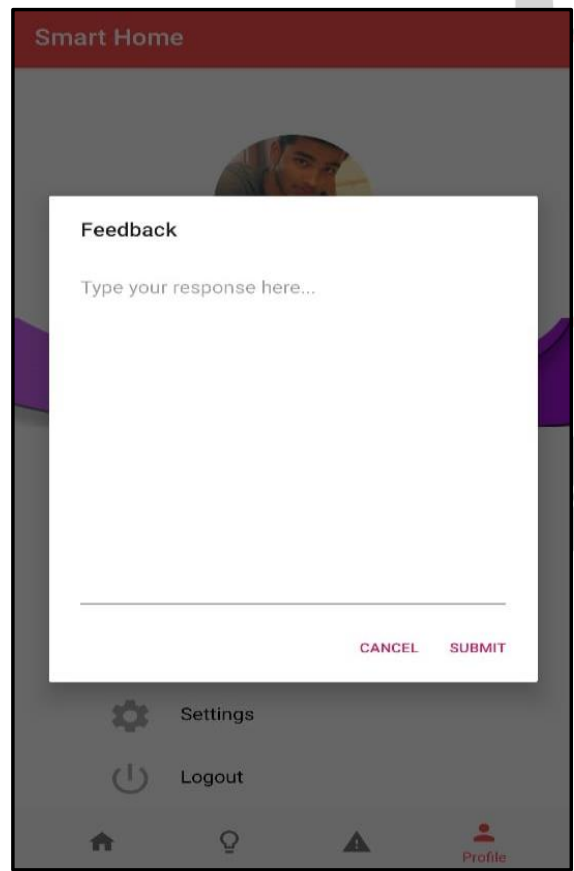

Figure.21: Dialog for response

Whenever the user wants to write any feedback, request or report any problem the dialog as shown in figure. 21 appears to the user to fill in the response.

The project also includes a website part dedicated to the admin where the admin has its own dashboard where the admin can be able to see if any request is made by a user or the problems which are faced by the user when using the device. The user can also give their feedback which can be seen by the admin in his website. Below are some screenshots of the admin website.

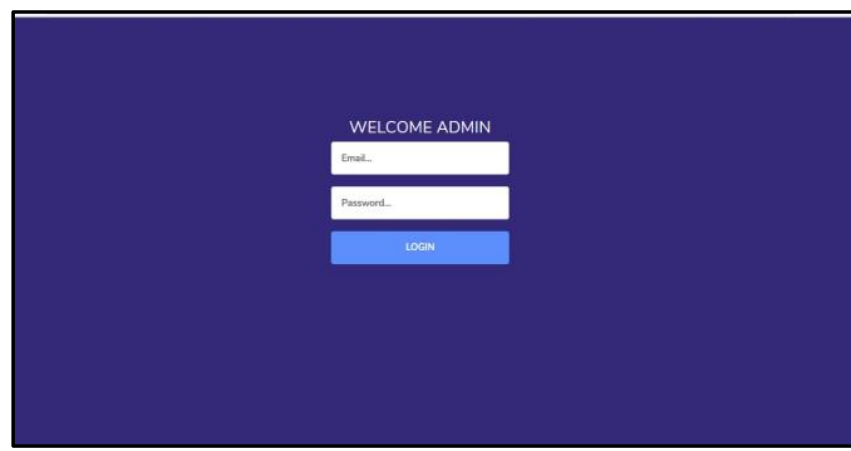

Figure. 22: Admin Login Page

The above interface is the admin login page where the admin needs to enter its credentials in order to enter the website. The credentials are already stored in the database, these credentials are validated from the database.

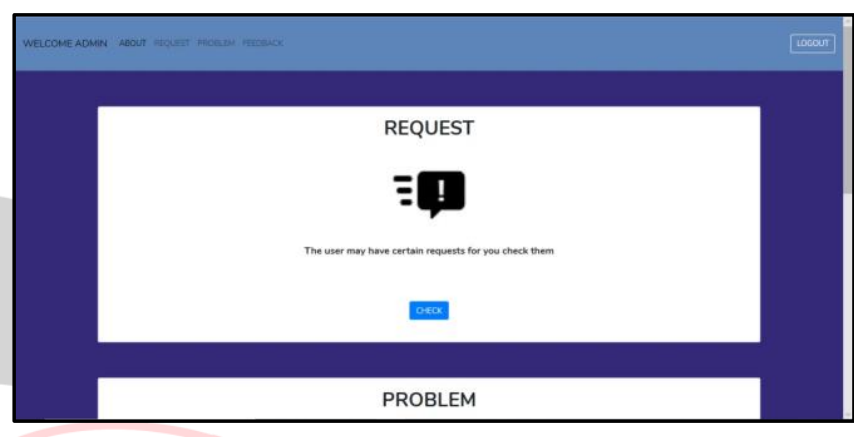

Figure.23: Admin Interface

The above figure is the admin interface where the admin can see the request, problem and feedback pages. Through this interface the admin can view either of the following requests, problems or feedback.

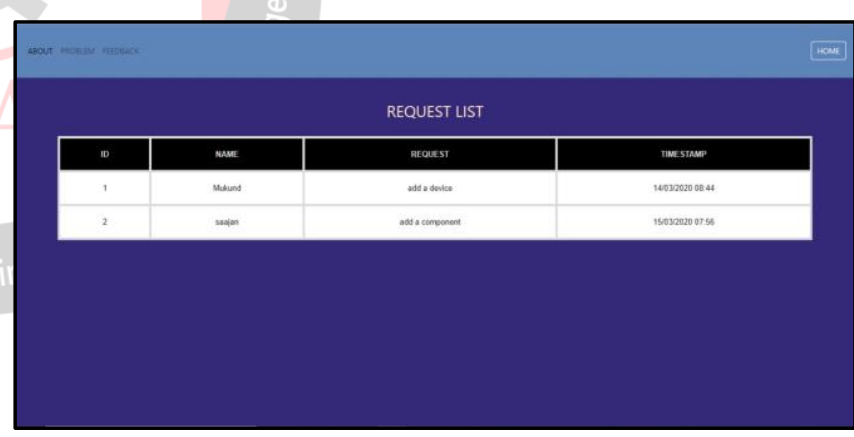

Figure.24: Request Page

The above interface is the request page for the admin to view all the requests made by the user. The user with the help of the android application can send its request to the admin. These requests are stored in the database and through the website the admin can fetch all the information.

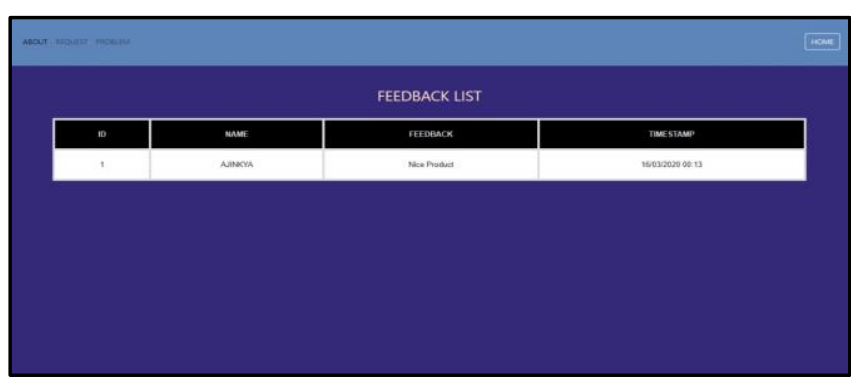

Figure.25: Feedback page 
The above interface is the feedback page for the admin to view all the feedback made by the user. The user can give its opinion about the system through the android application. The admin through the website fetches the feedback which is stored in the database through the application.

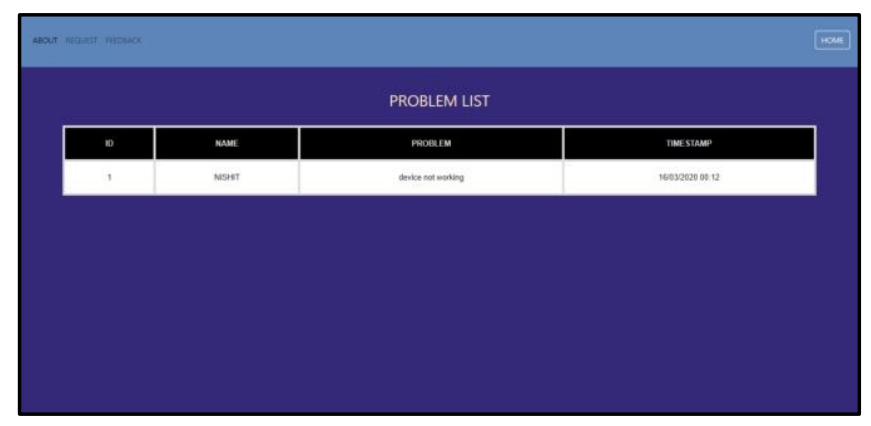

Figure.26: Problem page

The above interface is created for the admin to view all the problems faced by the user while using the system. The user may face certain issues with the device which the user needs to convey to the admin. Hence this page will help the admin to keep a look at all the problems faced by the user.

\section{CONCLUSION}

A Smart Home is a system of internet-connected devices and appliances. It allows homeowners to manage and monitor their home more efficiently. Smart homes are always accompanied with smart devices, but they are not limited to just smart devices. In this paper, we have discussed how we can control appliances remotely which cannot connect to the internet directly, with the help of IFTTT and any MQTT broker we can use our google assistant to control any appliances. Analyzing our home based on various parameters is another important aspect which we addressed in our paper. Analysis of any parameter helps us to quantify the impact it has in our life. With the data being uploaded by our sensors to the database, our application provides the analysis by using various graphical methods. Given the climate change and pollution the world is witnessing it's very important that every house analyzes their house based on some important parameters like temperature, humidity and air-quality. The scope of this research is limitless. This research can be further carried on automating appliances according to the house ambience, reducing the system complexity and providing users suggestions on conserving energy and environment.

\section{ACKNOWLEDGMENT}

A few sublime human experiences defy expressions of any kind, and a feeling of true gratitude is one of them. We, therefore, are short of words to express our gratitude to our Project Guide Dr. Vinayak D. Shinde for his principled guidance, sharing his experience and providing upright help throughout this work. Their experience in this field and his solutions provided us to improve the quality of the project. This experience gave us encouragement and was the prime source to complete the project.

\section{REFERENCES}

[1] V. Suma, R. R. Shekar and K. A. Akshay, "Gas Leakage Detection Based on IOT," 2019 3rd International conference on Electronics, Communication and Aerospace Technology (ICECA), Coimbatore, India, 2019, pp. 1312-1315.

[2] K. C. Sahoo and U. C. Pati, "IoT based intrusion detection system using PIR sensor," 2017 2nd IEEE International Conference on Recent Trends in Electronics, Information \& Communication Technology (RTEICT), Bangalore, 2017, pp. 1641-1645

[3] S. Sarkar, S. Gayen and S. Bilgaiyan, "Android Based Home Security Systems Using Internet of Things(IoT) and Firebase," 2018 International Conference on Inventive Research in Computing Applications (ICIRCA), Coimbatore, 2018, pp. 102-105.

[4] H. Singh, V. Pallagani, V. Khandelwal and U. Venkanna, "IoT based smart home automation system using sensor node," 2018 4th International Conference on Recent Advances in Information Technology (RAIT), Dhanbad, 2018, pp. 1-5.

[5] W. Li, C. Yen, Y. Lin, S. Tung and S. Huang, "JustIoT Internet of Things based on the Firebase real-time database," 2018 IEEE International Conference on Smart Manufacturing, Industrial \& Logistics Engineering (SMILE), Hsinchu, 2018, pp. 43-47.

[6] K. Deve, G. Hancke and B. Silva, "Design of a smart fire detection system," IECON 2016 - 42nd Annual Conference of the IEEE Industrial Electronics Society, Florence, 2016, pp.6205-6210.

[7] H. V. Bhatnagar, P. Kumar, S. Rawat and T. Choudhury, "Implementation model of Wi-Fi based Smart Home System," 2018 International Conference on Advances in Computing and Communication Engineering (ICACCE), Paris, 2018, pp.23-28.

[8] P. V. Dudhe, N. V. Kadam, R. M. Hushangabade and M. S. Deshmukh, "Internet of Things (IOT): An overview and its applications," 2017 International Conference on Energy, Communication, Data Analytics and Soft Computing (ICECDS), Chennai, 2017, pp. 2650-2653.

[9] H. Durani, M. Sheth, M. Vaghasia and S. Kotech, "Smart Automated Home Application using IoT with Blynk App," 2018 Second International Conference on Inventive Communication and Computational Technologies (ICICCT), Coimbatore, 2018, pp. 393-397.

[10] A. Oak and R. D. Daruwala, "Assessment of Message Queue Telemetry and Transport (MQTT) protocol with Symmetric Encryption," 2018 First International Conference on Secure Cyber Computing and Communication (ICSCCC), Jalandhar, India, 2018, pp. 5-8, doi: 10.1109/ICSCCC.2018.8703314.

[11] G. M. Salim, H. Ismail, N. Debnath and A. Nadya, "Optimal light power consumption using LDR sensor," 2015 IEEE International Symposium on Robotics and Intelligent Sensors (IRIS), Langkawi, 2015, pp. 144-148, doi: 10.1109/IRIS.2015.7451601. 